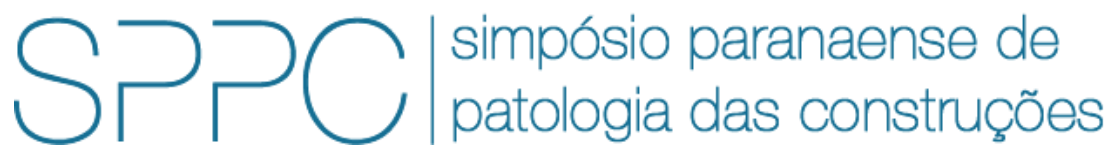

ISSN 2526-7248 artigo 2SPPC1028, pp. 344-353, 2017

\title{
Modelo matemático de ranqueamento: Avaliação do acréscimo do esforço horizontal nos pilares devido ao acúmulo de detritos
}

\author{
Cezar Falavigna ${ }^{1}$, André Luiz Tonso Fabiani ${ }^{2}$ \\ 1 Discente de Engenharia Civil, Universidade Federal do Paraná, cezarfalavigna@gmail.com \\ 2 Professor Doutor, Universidade Federal do Paraná, andre.dhs@ufpr.br
}

Resumo: O principal problema que uma obra de arte especial (OAE) pode apresentar seria sua ruína. Para o caso de pontes, na grande maioria dos casos dessas situações, o colapso é decorrente de fatores ligados ao corpo hídrico que essas tranpõem. Portanto, é indispensável gerar um modelo sobre o qual fatores ligados a parte hídrica possam ser avaliados. Um desses fatores é o incremento dos esforços nos pilares por conta do acúmulo de detritos. Utilizando o modelo de avaliação de obras de arte especiais (OAE) desenvolvido pelo Escritório Modelo de Engenharia Civil (EMEA) da Universidade Federal do Paraná (UFPR), esse artigo propõe uma metodologia de avaliação do quesito e como parametrizá-lo no modelo matemático do EMEA/UFPR.

Palavras-chave: Obras de arte especiais, Pontes, Pilares, Detritos, Modelo Matemático.

Abstract: The worst scenario that can happen to a bridge is its failure. When that situation occurs, most of them are caused by hydraulic problems. Therefore, it is important to generate an evaluation model that has on its analysis the problems caused by the river water flow. One of these, is the increase of pressure on piers due to the accumulation of debris. Using a bridge evaluation model developed by the Civil Engineering Model Office (EMEA) from the Paraná State Federal University (UFPR), this article proposes an evaluation methodology for the increase of pressure due to accumulation of debris and how to apply it on the mathematical model from EMEA/UFPR

Keywords: Bridges, Piers, Debris, Mathematical model, Evaluation model. 
FALAVIGNA, C.; FABIANI, A. L. T., MODELO MATEMÁTICO DE RANQUEAMENTO: AVALIAÇÃO DO ACRÉSCIMO DO ESFORÇO HORIZONTAL NOS PILARES DEVIDO AO ACÚMULO DE DETRITOS. $2^{\circ}$ Simpósio Paranaense de Patologia das Construções (2 SPPC), artigo 2SPPC1028, pp. 344-353, 2017. DOI: 10.4322/2SPPC.2017.028

\section{Introdução}

O maior problema que pode ocorrer com uma ponte após sua concepção, projeto e construção seria seu colapso. A falta dessa obra de arte especial (OAE) na malha rodoviária ou ferroviária pode fazer viagens ficarem mais longas, custos mais caros ou mesmo isolar uma determinada região de receber bens e serviços.

Um estudo realizado por Wardhana e Hadipriono [1] envolvendo cerca de 500 obras que entraram em colapso nos Estados Unidos entre 1989 e 2000 notou que quase $53 \%$ dos colapsos foram por inundações e erosão. Itens como sobrecarga e impacto de embarcações, caminhões ou trens somaram 20\%. Chang [2] realizou um estudo envolvendo 383 falhas, dos quais $24,5 \%$ foram relacionados aos pilares e $71,8 \%$ aos encontros. As causas dos colapsos foram divididas em 38,8\% para deficiências no canal, $29,6 \%$ à mudança vigorosa do escoamento, $20,0 \%$ ocasionado por detritos e somente $4,7 \%$ decorrentes de problemas estruturais.

Por essas razões, analisar a vulnerabilidade das pontes em relação à parte hidráulica deve ser desenvolvido. Porém, olhar somente esse aspecto ignorando os demais, seria incorrer em uma avaliação da qual se excluiria todos os campos envolvidos na área e continuar a olhar as obras sob um aspecto. Então, incluir a análise em um modelo que enumera holisticamente a estrutura é essencial. $\mathrm{O}$ modelo matemático escolhido foi o desenvolvido pelo Escritório Modelo de Engenharia Civil - EMEA da Universidade Federal do Paraná.

\section{Metodologia}

\subsection{Modelo matemático EMEA/UFPR}

O modelo desenvolvido no EMEA/UFPR foi baseado no COST 345 [3] do CEB (Comité Euro-Internacional du Béton) e já é empregado na Eslovênia e Áustria. Ele consiste em ponderar sobre os seguintes parâmetros, dano, elemento estrutural, intensidade do dano, extensão da propagação do dano nos elementos e urgência de reparo. A formulação é descrita a seguir:

$$
R=\sum V_{d}=\sum B_{i} * K_{1 i} * K_{2 i} * K_{3 i} * K_{4 i}(1)
$$

$\mathrm{Vd}=$ valor do dano;

$\mathrm{Bi}=$ valor associado ao tipo de dano "i" sobre a segurança e/ou durabilidade;

$\mathrm{K} 1 \mathrm{i}$ = fator do elemento da estrutura, em função da sua importância no contexto da estrutura como um todo;

$\mathrm{K}_{2 \mathrm{i}}=$ fator indicativo da intensidade do tipo de dano "i”;

$\mathrm{K} 3 \mathrm{i}$ = fator relativo à extensão da propagação do tipo de dano "i" nos elementos inspecionados

K4i = fator enfatizante da urgência de intervenção para o dano "i”. 
FALAVIGNA, C.; FABIANI, A. L. T., MODELO MATEMÁTICO DE RANQUEAMENTO: AVALIAÇÃO DO ACRÉSCIMO DO ESFORÇO HORIZONTAL NOS PILARES DEVIDO AO ACÚMULO DE DETRITOS. $2^{\circ}$ Simpósio Paranaense de Patologia das Construções (2 SPPC), artigo 2SPPC1028, pp. 344-353, 2017. DOI: 10.4322/2SPPC.2017.028

Cada um desses parâmetros possue um intervalo de valores nos quais se encaixam, descritos no "Bulletin" 243 [4] do CEB e resumido para cada índice na tabela 1, a seguir:

Tabela 1: Intervalo de valores dos parâmetros do modelo

\begin{tabular}{cc}
\hline Parâmetro & Intervalo \\
\hline B - tipo de dano (manifestação patológica) & 1,00 a 5,00 \\
K1 - fator do elemento da estrutura & 0,10 a 0,60 \\
K2 - fator indicativo da intensidade do dano & 0,00 a 2,00 \\
K3 - fator relativo à extensão da propagação do dano & 0,50 a 2,00 \\
K4 - fator enfatizante da urgência da intervenção & 1,00 a 5,00 \\
\hline
\end{tabular}

Como se pode notar, as amplitudes são diferentes para cada parâmetro, por exemplo, no caso de B (manifestação patológica), o valor varia conforme a severidade da manifestação, ou seja, estética causando insegurança ao usuário possui um valor menor que corrosão da armadura com desplacamento e rompimento de barra. Com esse mesmo princípio, o fator do elemento da estrutura (K1), também varia, já que em caso de falha de alguns elementos podem causar a ruína da obra, quando outros não terão o mesmo impacto. É o caso da ruptura de um pilar ou viga comparado à ruptura de um guarda-corpo.

Já o fator de intensidade do dano (K2) representa com qual gravidade a manifestação aparece no elemento já que apenas afirmar que existe corrosão da armadura com desplacamento não indica a magnitude que isso se desponta. Entretanto, somente a intensidade não é suficiente, pois essa degradação pode estar localizada ou espalhada no elemento da estrutura, para tal existe o fator relativo à extensão da propagação do dando (K3). O último fator é associado à urgência de intervenção (K4), esse parâmetro cabe ao engenheiro inspetor atribuir conforme as condições existentes.

Todavia, considerar somente o valor oriundo desses parâmetros não é suficiente para ser possível comparar estruturas, isto porque no caso de pontes maiores estas provavelmente possuem mais manifestações que pontilhões (travessia com até 6 metros) e isso faz com que seu valor de dano seja maior, considerando uma a mesma morfologia para ambas. Para o caso de estruturas com morfologias diferentes essa questão é agravada, pois como os elementos mudam, isso resulta novos parâmetros $\mathrm{K} 1$, o que gera resultados mais diversos que o caso anterior.

Para sanar esse problema, a nota obtida pelos indicadoress deve ser divida por um valor de referência, assim é possível obter um índice de performance. $O$ valor de referência é atribuído a todos os danos que podem ocorrer na estrutura, multiplicados pelos valores máximos de intenside $(\mathrm{K} 2=\mathrm{K} 3=2,00)$ e extensão e pelo valor unitário de urgência $(K 4=1,00)$. $O$ índice de performance é equacionado a seguir:

$$
I_{p}=\frac{\sum V_{d}}{\sum V_{d, r e f}}(2)
$$


Onde:

Ip = índice de performance;

$\mathrm{Vd}=$ valor do dano; e

Vd,ref $=$ valor de referência .

Explanado sobre o modelo de avaliação desenvolvido no EMEA/UFPR, é necessário então incrementar a análise hídrica nesse. Esta é importante ser analisada em três partes, se o gabarito é suficiente para o escoamento do rio, se a erosão nos pilares não atinge níveis preocupantes e o objeto deste artigo se 0 incremento da força horizontal nos pilares em decorrência do acúmulo de detritos está em um nível alarmante.

\subsection{Análise da segurança hidráulica no modelo EMEA/UFPR}

Como citado anteriormente, a avaliação dos quesitos hidráulicos será feita em 3 partes, porém nesse artigo só será relatado o incremento de força horizontal nos pilares. Porém, para o modelo EMEA, todas três análises terão em comum 0 elemento estrutural da ponte (K1) a segurança hídrica, ou seja, o K1 sempre será igual a 0,40. Portanto cada avaliação possuirá um B próprio, o K1 em comum, e K2, K3, K4 com mensurações exclusivas para cada dano.

Inicialmente será descrito como é feita a ponderação da análise do esforço horizontal nos pilares e o embasamento teórico para se chegar a tal, em seguida, é explanado como aplicar o diagnóstico no modelo matemático do EMEA/UFPR.

\subsubsection{Acréscimo de esforço horizontal nos pilares devido ao acúmulo de detritos}

O esforço horizontal é orindo da pressão da água no pilar, porém quando existe uma aglomeração de material na sua face, isso incorre em uma força resultante não prevista em projeto. Para o cálculo do esforço a NBR 7187:2003 - Projetos de ponte de concreto armado e concreto protendido [5] - recomenda que "a pressão da água em movimento sobre os pilares e elementos da fundação pode ser determinada através da expressão" (Eq. 3):

$$
p=k \cdot v^{2}
$$

$\mathrm{p}=$ pressão estática equivalente, em $\mathrm{kN} / \mathrm{m}^{2}$;

$\mathrm{v}=$ velocidade da água em $\mathrm{m} / \mathrm{s} ; \mathrm{e}$

$\mathrm{k}=$ coeficiente dimensional conforme seção do pilar.

O coeficiente dimensional - $\mathrm{k}$ - varia de acordo com a seção do pilar, se é um círculo ou um quadrilátero, e a direção do escoamento em relação à forma. Porém, usualmente, a força resultante do escoamento sobre qualquer forma é dada pela expressão (Ep. 4) a seguir, que efetivamente será usada para o modelo do EMEA/UFPR: 


$$
F=C_{d} \cdot \rho \cdot A \cdot \frac{u^{2}}{2}(4)
$$

$\mathrm{F}=$ força resultante, em $\mathrm{N}$;

$\mathrm{Cd}=$ coeficiente de arresto, adimensional;

$\rho=$ massa específica do fluido, em $\mathrm{kg} / \mathrm{m}^{3}$;

$\mathrm{A}=$ área do pilar submersa, em $\mathrm{m}^{2} ; \mathrm{e}$

$\mathrm{u}=$ velocidade média do escoamento, em $\mathrm{m} / \mathrm{s}$.

A princípio pode-se crer que são duas expressões diferentes, no entanto essas são a mesma, como demonstrado a seguir (Eq. 5, 6 e 7):

$$
\begin{gathered}
p=\frac{F}{A}(5) \\
p=C_{d} \cdot \rho \cdot \frac{u^{2}}{2}(6) \\
k \cdot v^{2}=C_{d} \cdot \rho \cdot \frac{u^{2}}{2}(7)
\end{gathered}
$$

As velocidades de escoamento são representadas por $v$ na equação 3 , oriunda da norma e u na equação 4, comumente utilizada na mecânica dos fluidos, ambas em $\mathrm{m} / \mathrm{s}$. Além disso, o $\mathrm{k}$ na expressão é dado em $\mathrm{kN} / \mathrm{m}^{2}$ enquanto a equação 4 , utilizando $\mathrm{C}_{\mathrm{d}}$, usa como unidades $\mathrm{N} / \mathrm{m}^{2}$. Portanto, é necessário compatibilizar unidades, para isso precisa-se dividir por 1000, resultanto em (Eq. 8):

$$
k=\frac{C_{d} \cdot \rho}{2} \cdot \frac{1}{1000}(8)
$$

A massa específica da água (p) é $1000 \mathrm{~N} / \mathrm{m}^{2}$, procedendo em (Eq. 9 e 10):

$$
\begin{aligned}
k & =\frac{C_{d}}{2}(9) \\
C_{d} & =2 k(10)
\end{aligned}
$$

Portanto, no método de análise para a segurança hídrica - força horizontal nos pilares - os valores para $C_{d}$ serão o dobro dos encontrados em norma. Porém por experiência em inspeções de pontes, é comum encontrar-se pilares octogonais, não contemplados em norma. Para se estabelecer esse valor foram utilizadas relações de área comum entre o círculo-octógno e quadriláterio-octógono, e também o caminho até que ocorra a separação do escoamento na forma, com isso foi obtido o valor de $\mathrm{k}$ igual a 0,47 e, portanto, um $\mathrm{Cd}$ de 0,94.

A figura a seguir (Fig. 1) ilustra todos os valores conforme o ângulo de incidência: 
FALAVIGNA, C.; FABIANI, A. L. T., MODELO MATEMÁTICO DE RANQUEAMENTO: AVALIAÇÃO DO ACRÉSCIMO DO ESFORÇO HORIZONTAL NOS PILARES DEVIDO AO ACÚMULO DE DETRITOS. $2^{\circ}$ Simpósio Paranaense de Patologia das Construções (20 SPPC), artigo 2SPPC1028, pp. 344-353, 2017. DOI: 10.4322/2SPPC.2017.028

\begin{tabular}{|c|c|c|c|}
\hline Representação & $\begin{array}{l}\text { Ängulo de } \\
\text { Incidência }\end{array}$ & K & $\mathrm{C}_{\mathrm{d}}$ \\
\hline & $90^{\circ}$ & 0,71 & 1,42 \\
\hline & $45^{\circ}$ & 0,54 & 1,08 \\
\hline & $0^{\circ}$ & 0,00 & 0,00 \\
\hline & $0^{\circ}-90^{\circ}$ & 0,34 & 0,68 \\
\hline & $0^{\circ}-90^{\circ}$ & 0,47 & 0,94 \\
\hline
\end{tabular}

Figura 1: Valores de k e $C_{d}$ para as seções dos pilares. Fontes: Os autores.

Com o acréscimo dos detritos, a área na formulação irá aumentar, consequentemente, a força horizontal também. Devido à cor e turbidez das águas dos rios, não é possível saber quanto dos detritos está submerso, por isso, admitese a simplicação de que o material que está acima do nível d'água também está abaixo. Conforme ilustrado abaixo (Fig. 2):

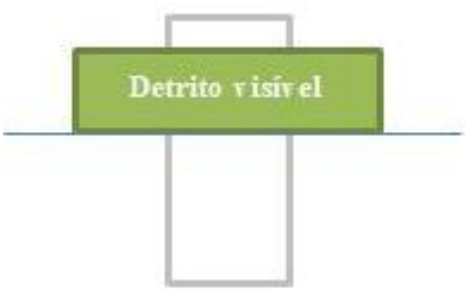

Visto em campo

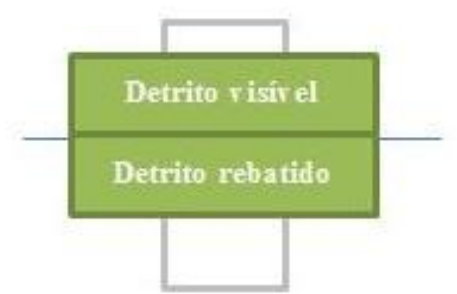

Espelhado - simplificação

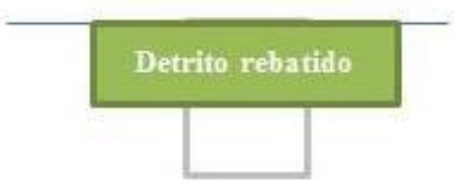

Área para o cálculo

Figura 2: Simplificação dos detritos. Fonte: Os autores.

Definido a formulação e como avaliar os itens dessa, falta definir os parâmetros para o modelo EMEA/UFPR. O primeiro é o valor para B - manifestação patológica, igual a 3,00. Esse número é decorrente da comparação com valores de outros danos utilizados no modelo. As manifestações utilizadas foram "Dano não gerando esforços imprevistos" cujo B vale 2,00 e "Dano gerando esforços imprevistos" cujo B vale 4,00. A partir da média de ambos, é possível chegar ao valor adotado de 3,00. O valor foi adotado, pois como a escala é de 1,00 até 5,00, um peso de 4,00 representaria $80 \%$ da escala de variação, o que foi considerado muito alto, já adotar 
FALAVIGNA, C.; FABIANI, A. L. T., MODELO MATEMÁTICO DE RANQUEAMENTO: AVALIAÇÃO DO ACRÉSCIMO DO ESFORÇO HORIZONTAL NOS PILARES DEVIDO AO ACÚMULO DE DETRITOS. $2^{\circ}$ Simpósio Paranaense de Patologia das Construções (20 SPPC), artigo 2SPPC1028, pp. 344-353, 2017. DOI: 10.4322/2SPPC.2017.028

2,00 teria o efeito inverso, seria somente $40 \%$ da graduação, o que seria muito pequeno para o dano. Portanto, B será igual a 3,00. Já o K1, como citado anteriormente, será sempre igual a 0,4, referente à segurança hídrica.

O próximo parâmetro é o K2, calculado utilizando a seguinte formulação (Eq.11):

$$
\% k 2=\frac{F_{\text {com detritos }}}{F_{\text {sem detritos }}}-1
$$

Onde:

F com detritos = força horizontal nos pilares com o acúmulo de detritos; e

F sem detritos = força horizontal nos pilares sem o acúmulo de detritos.

O valor resultante da equação 11 deve ser comparado com a Tabela 2, que representa os valores de $\mathrm{K} 2$ de acordo com a intensidade que a manifestação se apresenta:

Tabela 2: Intervalo de valores para o K2 - Intensidade do dano

\begin{tabular}{cccc}
\hline Tipo & Grau & Critério & Intervalo \\
\hline 0 & Insignificante & Dano irrelevante & 0,00 \\
I & Pequeno & $\begin{array}{c}\text { Dano pequeno, em menos de 10\% da área } \\
\text { total do elemento }\end{array}$ & 0,50 \\
II & Médio & $\begin{array}{c}\text { Dano intermediário, confinado em uma área } \\
\text { pequena de 10\% a 25\% da área total do } \\
\text { elemento }\end{array}$ & 1,00 \\
III & Grande & $\begin{array}{c}\text { Dano grande, de 25\% a 75\% da área total do } \\
\text { elemento, ou em muitos lugares de um } \\
\text { elemento estrutural }\end{array}$ & 1,50 \\
& Imenso & $\begin{array}{c}\text { Dano muito grande, na maior parte da área de } \\
\text { um elemento, mais de 75\% da área total do } \\
\text { elemento }\end{array}$ & 2,00 \\
\hline
\end{tabular}

No caso, o dano não seria medido em área e sim em quanto aumentou o esforço em relação ao que seria previsto pela norma. Na ocorrência de mais de um pilar com acúmulo de detritos, como simplicação e segurança, o pilar que apresentar a pior manifestação será a base de cálculo da intensidade do esforço.

O próximo parâmetro é a amplitude de propagação, K3, que é calculado pela simples relação entre o número de pilares com detritos e o número total de pilares imersos em água (Eq. 12). Dessa relação compare-se com a tabela 3 de extensão de propagação do dano.

$$
\% K 3=\frac{\text { Pilares na água com detritos }}{\text { Pilares na água }}
$$


FALAVIGNA, C.; FABIANI, A. L. T., MODELO MATEMÁTICO DE RANQUEAMENTO: AVALIAÇÃO DO ACRÉSCIMO DO ESFORÇO HORIZONTAL NOS PILARES DEVIDO AO ACÚMULO DE DETRITOS. $2^{\circ}$ Simpósio Paranaense de Patologia das Construções (2 SPPC), artigo 2SPPC1028, pp. 344-353, 2017. DOI: 10.4322/2SPPC.2017.028

Tabela 3: Intervalo de valores para o parâmetro K3 - extensão de propagação

\begin{tabular}{cc}
\hline Critério & K3 \\
\hline Dano aparece em menos de 10\% dos elementos estruturais da OAE & 0,50 \\
Dano aparece ente $10 \%$ e $25 \%$ dos elementos estruturais da OAE & 1,00 \\
Dano aparece entre $25 \%$ e $75 \%$ dos elementos estruturais da OAE & 1,50 \\
Dano aparece entre $75 \%$ e $100 \%$ dos elementos estruturais da OAE & 2,00 \\
\hline
\end{tabular}

Por último, os valores associados à urgência de intervenção são apresentados a seguir. O dano é classificado de acordo com a tabela 4, a partir da avaliação do inspetor e das condições existentes. Como fatores importantes de análise destas, recomenda-se ao avaliador observar se a estrutura é isostática ou hiperestática, o comprimento do vão, o quanto a seção é contraída com o acúmulo de materiais e se possível, comparar com dimensionamento estrutural do pilar, caso seja permitido o acesso ao projeto. A partir disso, pode-se atribuir a urgência de reparo, conforme ilustrado pela tabela 4 .

Tabela 4: Intervalo de valores para o parâmetro K4 - urgência de intervenção

\begin{tabular}{|c|c|c|}
\hline Classificação & Intervalo & K4 \\
\hline Não urgente & $\begin{array}{l}\text { Intervenção não urgente pois o dano não interfere na } \\
\text { utilização e capacidades da OAE, também não altera a } \\
\text { sua durabilidade }\end{array}$ & 1,00 \\
\hline Dano à reparar & $\begin{array}{c}\text { Dano deve ser reparado em período não maior que } 5 \\
\text { anos, para garantir a servicibilidade e não } \\
\text { compremeter a durabilidade }\end{array}$ & 2,50 \\
\hline Reparo imediato & $\begin{array}{c}\text { Reparo imediato, pois o dano já está comprometendo } \\
\text { a utilização da OAE, com risco às pessoas }\end{array}$ & 4,00 \\
\hline Limitação de carga & $\begin{array}{l}\text { Limitação de carga, interrupção de tráfego e } \\
\text { escoramento imediato deve ser feito, além do reparo }\end{array}$ & 5,00 \\
\hline
\end{tabular}

A descrição que se encontra no "Bulletin" 243 [4] do CEB para a limitação de carga (Tabela 4), afirma que deve ser feito o escoramento imediato, porém, no caso do acúmulo de detritos nos pilares, o escoramento não deve acontecer, a solução é a limpeza com urgência desses

Com todos os índices estabelecidos é possível calcular o valor do dano para o acréscimo do esforço horizontal nos pilares por conta de detritos presos, e assim agregar ao modelo EMEA/UFPR.

\section{Conclusões}

Aplicando o modelo desenvolvido pelo EMEA/UFPR, é possível perceber que ele analisa a obra de arte especial de maneira global em função do risco que a manifestação patológica representa para a estrutura. No entanto, estudos mostram que as principais razões para o colapso das pontes estão ligadas diretamente a parte hidríca do conjunto.

A análise anterior cobre um dos pontos desse aspecto, onde se avalia o acréscimo das forças horizontais. Compatibilizando com requisitos da NBR 7187:2003 com um 
FALAVIGNA, C.; FABIANI, A. L. T., MODELO MATEMÁTICO DE RANQUEAMENTO: AVALIAÇÃO DO ACRÉSCIMO DO ESFORÇO HORIZONTAL NOS PILARES DEVIDO AO ACÚMULO DE DETRITOS. $2^{\circ}$ Simpósio Paranaense de Patologia das Construções (2 SPPC), artigo 2SPPC1028, pp. 344-353, 2017. DOI: 10.4322/2SPPC.2017.028

modelo de análise do dano, é possível então referenciar os dados obtidos com a formulação desenvolvida pelo EMEA/UFPR. Assim mantém-se o padrão comparativo que esse permite entre obras de arte especiais.

A ferramenta descrita no artigo mostra-se aplicável em campo já que não necessita de muitas informações a serem coletadas, a não ser a profundidade do canal e a dimensão do pilar, que podem ser feitas fácilmente. Além disso, o instrumento começa a preencher a falha de que quando se faz a análise de uma ponte, 0 enfoque dado é somente à estrutura, porém como citado anteriormente, os colapsos desse quesito são bem menos recorrentes do que se comparada às que envolvem o curso d'água. Portanto, deve-se existir uma mudança de avaliação, ou melhor, um incremento nos procedimentos de inspeção, no qual, seja incluso uma ponderação sobre os efeitos gerados pelo acréscimo de esforço horizontal nos pilares devido aos detritos.

Esse enfoque não agregado, quando se trata de manutenção e conservação de obras de arte especiais, que como comprovado, faz parte dos grupos que mais ocasionam ruptura das estruturas. Logo, deve-se somar às inspeções de pontes avalições sobre o rio sobre o qual passam por cima, não mais entendendo somente como um obstáculo a ser transposto e sim como o principal gerador de colapsos nessas estruturas.

\section{Agradecimentos}

À Universidade Federal do Paraná, ao Setor de Tecnologia da UFPR, ao Departamento de Construção Civil (DCC) - UFPR, ao Departamento de Hidráulica e Saneamento (DHS) - UFPR, ao Programa de Pós-Graduação em Engenharia de Construção Civil (PPGECC), ao Programa de Pós-Graduação em Engenharia de Recursos Hídricos (PPGERHA), o Escritório Modelo de Engenharia Civil - EMEA da UFPR e principalmente ao professor PhD. Mauro Lacerda Santos Filho por propiciar as condições necessárias para que essa pesquisa fosse realizada.

\section{Referências}

[1] Wardahana. Kumalasari; Hadripriono, Fabian C. (2003). Analysis of recent bridge failures in the United States. Journal of Performance Of Constructed Facilities. 17:3 p. 144-150.

[2] Chang, F.F.M. (1973). A statistical summary of the cause and cost of bridge failures. Federal Highway Administration - FHWA emergency relief files.

[3] European co-operation in the field of scientific and technical research. (2007). COST 345: Methods used in the european states and assess the condition of highway structures. Disponível em: <http://cost345.zag.si/Reports/COST_345_WG23.pdf>. Acesso em 21 ago. 2016

[4] Comité euro-internacional du betón. (1998). Bulletin 243: Strategies for Testing and Assessment of Concrete Structures. Suíça. 
FALAVIGNA, C.; FABIANI, A. L. T., MODELO MATEMÁTICO DE RANQUEAMENTO: AVALIAÇÃO DO ACRÉSCIMO DO ESFORÇO HORIZONTAL NOS PILARES DEVIDO AO ACÚMULO DE DETRITOS. $2^{\circ}$ Simpósio Paranaense de Patologia das Construções ( $2^{\circ}$ SPPC), artigo 2SPPC1028, pp. 344-353, 2017. DOI: 10.4322/2SPPC.2017.028

[5] Abnt - Associação Brasileira de Normas e Técnicas: NBR 7187:2003 - Projeto de pontes de concreto armado e de concreto protendido - Procedimento. 